\title{
Neuroendocrine Cell Hyperplasia of Infancy
}

National Cancer Institute

\section{Source}

National Cancer Institute. Neuroendocrine Cell Hyperplasia of Infancy. NCI Thesaurus.

Code C120169.

A form of interstitial lung disease characterized by increased numbers of pulmonary neuroendocrine cells, typically presenting in the first year of life with persistent tachypnea, retractions, crackles and hypoxemia that has a highly specific chest CT pattern that includes ground-glass opacities and air-trapping. 